\title{
Optical and magneto-optical properties of ferromagnetic full-Heusler films: experiments and first-principles calculations
}

\author{
F. Ricci ${ }^{1}$, S. Picozzi ${ }^{2,1}$, A. Continenza ${ }^{1,3}$, F. D’Orazio ${ }^{1,3}$, F. Lucari ${ }^{1,2}$, \\ K. Westerholt ${ }^{4}$, M. Kim ${ }^{5, *}$ and A. J. Freeman ${ }^{5}$ \\ 1 Dip. di Fisica, Univ. dell'Aquila, 67010 Coppito (L'Aquila), Italy; \\ 2 Istituto Nazionale di Fisica della Materia (INFM-CNR); \\ 3 Consorzio Nazionale Interuniversitario Scienze della Materia (CNISM); \\ 4 Institut fur Experimentalphysik IV, Ruhr-Univ. Bochum, Germany; \\ 5 Dept. of Physics and Astronomy, \\ Northwestern Univ., 60208 Evanston, Il, USA
}

\begin{abstract}
We report a joint theoretical and experimental study focused on understanding the optical and magneto-optical properties of Co-based full-Heusler compounds. We show that magneto-optical spectra calculated within ab-initio density functional theory are able to uniquely identify the features of the experimental spectra in terms of spin resolved electronic transitions. As expected for $3 d$-based magnets, we find that the largest Kerr rotation for these alloys is of the order of $0.3^{\circ}$ in polar geometry. In addition, we demonstrate that $(i)$ multilayered structures have to be carefully handled in the theoretical calculations in order to improve the agreement with experiments, and $(i i)$ combined theoretical and experimental investigations constitute a powerful approach to designing new materials for magneto-optical and spin-related applications.

PACS numbers: 75.50.Cc, 78.20.Ls, 71.15.-m
\end{abstract}

\footnotetext{
* Present address: Seoul National University, Seoul, Korea
} 


\section{INTRODUCTION}

Spin electronics, or spintronics, is a multidisciplinary field that involves the active manipulation of the electron spin degree of freedom. Recent developments in material design allow to combine magnetic and semiconducting character, thus opening the possibility to build devices with highly polarized currents. In fact, one of the major challenges in spintronics is the search for compounds that could act as efficient injectors of spin-polarized current in semiconductors. Diluted magnetic semiconductors (DMS) 1 are obviously among the best candidates as spin-polarized sources, due to their excellent structural compatibility with the spin-current semiconducting "sink". Indeed, spin-light emitting devices have been successfully realized using DMS as spin-injectors ${ }^{2}$. However, the very low Curie temperatures and the need for high magnetic fields seem to have hindered so far a large-scale application of DMS in the spin-injection framework. On the other hand, the discovery of a wide class of half-metallic (HM) compounds, initially predicted by de Groot et al. $\underline{3}$, has gained importance in this context $\stackrel{4}{*}$. The $\mathrm{Co}_{2} \mathrm{MnX}$ (with $\mathrm{X}=\mathrm{Al}, \mathrm{Si}, \mathrm{Ga}$, Ge, Sn) full-Heusler family has interested the scientific community because of its ideal $100 \%$ spin polarization at the Fermi level, a good structural matching with mainstream semiconductors (in terms of lattice constants and crystal structure) and very high Curie temperatures $\underline{5} \underline{\underline{5}}(\sim 900 \mathrm{~K})$.

Recently, a number of Heusler compounds have been grown epitaxially on semiconducting substrates: for example, $\mathrm{Co}_{2} \mathrm{MnGe}^{7}, \mathrm{Co}_{2} \mathrm{MnSi} \stackrel{\text { ‥9 }}{ }, \mathrm{Ni}_{2} \mathrm{MnGa}^{10}, \mathrm{Ni}_{2} \mathrm{MnGe}^{11}$ on $\mathrm{GaAs}$ [001] and $\mathrm{Ni}_{2} \mathrm{MnIn}$ on InAs $[001]^{12}$. On the other hand, also RF-sputtering technique has been

shown to produce homogeneous Heusler films with good structural and magnetic quality $\underline{13}, 14$. The first noticeable application of electrical spin injection from $\mathrm{Co}_{2} \mathrm{MnGe}$ into a $p-i-n$ $\mathrm{Al}_{0.1} \mathrm{Ga}_{0.9} \mathrm{As} / \mathrm{GaAs}$ light emitting diode heterostructure was reported only recently $\underline{\underline{15}}$ : a steady state polarization of $13 \%$ resulted at $2 \mathrm{~K}$. The injected polarization was significantly lower than the theoretically predicted $100 \%$ value expected for half-metals 6.16 , pointing to the importance of interfacial disorder or temperature effects $\frac{17}{}$.

Moreover, within this family of compounds, $\mathrm{PtMnSb}$ has been found to exhibit a giant Kerr rotation (up to $2.0^{\circ}$ at room temperature and $5.0^{\circ}$ at $80 \mathrm{~K}$ ) that is totally unexpected for a $3 d$-based material ${ }^{18}$. This is of technologically relevant importance in the context of magneto-optical reading and recording technology. Since then, there has been great interest in magneto-optics for this class of compounds $\frac{19}{}$; in particular, $\mathrm{Co}_{2} \mathrm{MnSi}^{20}$ and 
$\mathrm{Co}_{2} \mathrm{MnGe}^{21}, 22.23$ were characterized by means of X-ray absorption and magnetic circular dichroism. However, to our knowledge, there are no experimental or first-principles characterizations (within the local-spin density approximation (LSDA) to density functional theory (DFT)) of these Co-based compounds, as far as the magneto-optical Kerr effect (MOKE) is concerned.

This paper presents a joint theoretical and experimental study of Heusler compounds, aimed at a more complete understanding of their optical and magneto-optical properties. Therefore, we pick two prototype examples of the full-Heusler family $\left(\mathrm{Co}_{2} \mathrm{MnGe}\right.$ and $\mathrm{Co}_{2} \mathrm{MnSn}$ ), which are similar enough to support the comparison between calculated and experimental MOKE spectra. These crystals are expected to have a high degree of spinpolarization at the Fermi level $(\sim 100 \%)$, and a similar response when they are excited with electromagnetic radiation.

The paper is organized as follows: after some computational details are reported in Sec. II, we focus on the description of the experimental approach to the magneto-optical properties (Sec. III) and on the comparison between theory and experiments for the Kerr rotation and ellipticity (Sec. IV). The good agreement with the experimental spectra suggests that a first principles calculation describes sufficiently well the Heusler's electronic structure on the basis of a bare DFT approach, without invoking correlation or excited-state effects beyond a standard LSDA treatment. Therefore, in Sec. V we give a comprehensive theoretical description of both optical and magneto-optical properties for $\mathrm{Co}_{2} \mathrm{MnGe}$ and $\mathrm{Co}_{2} \mathrm{MnSn}$ on the basis of their underlying electronic structure. In Sec. VI we draw conclusions.

\section{COMPUTATIONAL DETAILS}

Accurate first-principles calculations, using the full-potential linearized augmented planewave (FLAPW) method $\underline{24}$, were performed within the LSDA approximation to DFT, according to the von Barth-Hedin treatment for the exchange-correlation functional. In the calculations, we used muffin tin radii of 2.1 a.u. for Ge, Co, Mn and 2.3 a.u. for Sn; wave function and charge density cut-offs were set at 3.8 and 8.0 a.u., respectively and 110 kpoints were used in the irreducible wedge of the Brillouin zone for the self-consistent cycle, according to the standard Monkhorst-Pack procedure ${ }^{25}$.

We recall that MOKE is based on the change experienced by linearly polarized light 
reflected from a ferromagnetic material, which becomes elliptically polarized with its major axis rotated by an angle $\theta_{\kappa}$ (with respect to the incident polarization plane) and ellipticity $\varepsilon_{\kappa}{ }_{\kappa}^{26}$. The MOKE interband transition is a direct consequence of the interplay between spinorbit coupling and the exchange interaction: the relation between the magnetic field, acting on spins, and radiation, which interacts with the orbital degrees of freedom, is established through spin-orbit coupling. The symmetry breaking resulting from this interaction changes the electronic selection rules of the system in such a way that most of the important features in the conductivity tensor $\sigma(\omega)$ can be interpreted in terms of the bare band structure (see Sec. VD).

The optical conductivity tensor $\sigma$ is calculated within linear response theory in the long wavelength limit, using the relativistic single-particle Kohn-Sham eigenvalues and wave functions $^{27}$. Linear response theory within the Kubo formalism allows us to write the conductivity tensor $\sigma$ as:

$$
\sigma_{\lambda \lambda^{\prime}}(\omega)=\frac{i e}{m^{2} \hbar V} \sum_{(i \vec{k})_{o c c}}^{(j \vec{k})_{n o c c}} \frac{1}{\omega_{i j}}\left[\frac{\Pi_{i j}^{\lambda} \Pi_{j i}^{\lambda^{\prime}}}{\omega-\omega_{i j}+i / \tau}-\frac{\Pi_{i j}^{\lambda} \Pi_{j i}^{\lambda^{\prime}}}{\omega+\omega_{i j}+i / \tau}\right]
$$

where the sum is taken between the occupied and unoccupied states, $i, j$ respectively; $\Pi_{i j}^{\lambda}$ is the $\lambda$ component $(\lambda=x, y, z)$ of the dipole matrix calculated between the $i, j$ states; $\tau$ is the interband relaxation time. In polar geometry at normal incidence (i.e. with the propagation direction ( $z$ axis) parallel to the magnetization direction and perpendicular to the surface), for cubic crystals, the complex polar Kerr angle can be expressed to first order as:

$$
\Phi_{\kappa}=\theta_{\kappa}+i \varepsilon_{\kappa}=\frac{-\sigma_{x y}}{\sigma_{x x} \sqrt{1+i\left(4 \pi \sigma_{x x} / \omega\right)}}=\frac{-\sigma_{x y}}{D}
$$

where $\omega$ is the photon energy and we define the quantity $D$ containing only the diagonal terms of the optical conductivity. We account for other transitions that are not included in the interband term expressed in Eq. (11) (i.e. scattering at lattice imperfections or phonons $)^{28}$ by considering intraband transitions described by an empirical Drude contribution (characterized by an intraband relaxation time $\tau_{D}$ ) added to the diagonal elements of the $\sigma$ tensor:

$$
\sigma_{0}=\frac{\omega_{p}^{2}}{4 \pi\left(1-i \omega \tau_{D}\right)}
$$

Here the plasma frequency is expressed as:

$$
\omega_{p}=\frac{4 \pi e^{2}}{m^{2} V} \sum_{i \mathbf{k}, \lambda} \delta\left(\varepsilon_{i \mathbf{k}}-E_{F}\right)\left|\Pi_{i i}^{\lambda}\right|^{2}
$$


where $E_{F}$ is the Fermi energy, and $\Pi_{i i}^{\lambda}$ the diagonal element of the dipole matrix.

The calculation of the optical and magneto-optical properties has been performed using 1000 k-points. In order to consider the effect of finite lifetimes, as well as of the experimental resolution, a Lorentzian broadening equal to $\frac{\hbar}{\tau}=0.4 \mathrm{eV}$ was applied for both the interband and intraband contributions. Since MOKE is a very small effect, an accurate all-electron code with no approximations on the potential, as the FLAPW used in the present work, is highly recommended in order to obtain reliable results 29.

\section{EXPERIMENTAL RESULTS}

Experimental MOKE spectra were obtained on $\mathrm{Co}_{2} \mathrm{MnSn}$ and $\mathrm{Co}_{2} \mathrm{MnGe}$ films grown by RF-sputtering on $\mathrm{Al}_{2} \mathrm{O}_{3}$ a-plane substrates. Before deposition, a $5 \mathrm{~nm}$ thick seed layer of vanadium was deposited in order to induce excellent (110)-growth of the $100 \mathrm{~nm}$ thick Heusler film 13 . A final protective overlayer of amorphous alumina, $5 \mathrm{~nm}$ thick, was then grown on top of the film. At each photon energy complete hysteresis loops were performed and both $s$ - and $p$-waves MOKE rotation and ellipticity were measured as a function of the magnetic field, in the range \pm 5600 Oe. In this way, the presence of paramagnetic or diamagnetic terms can be detected as a linearly field dependent component and then subtracted from the overall signal. This is particularly important for low temperature measurements in which the cryostat windows may provide an appreciable diamagnetic contribution.

As expected in thin films, due to shape anisotropy, magnetization in the direction perpendicular to the surface is not (energetically) favored. This is evidenced by the linear field dependence of the polar MOKE hysteresis loops all over the magnetic field range (not shown). On the contrary, longitudinal s-polarized MOKE experiments lead to hysteresis loops with a saturation field on the order of 50 Oe.

As an example, we report in Fig. 1 some $s$-wave longitudinal MOKE hysteresis loops for the $\mathrm{Co}_{2} \mathrm{MnGe}$ sample at a photon energy of $0.83 \mathrm{eV}$. In-plane anisotropy is observed when longitudinal experiments are repeated with the sample rotated around the axis perpendicular to the surface; it turns out that the easy and hard axes are mutually perpendicular. The in-plane anisotropy is induced during growth by the step edges on the surface of the $\mathrm{Al}_{2} \mathrm{O}_{3}$

substrate. However, since we are interested in comparing the experimental MOKE results with the theoretical prediction, the meaningful quantity to be considered is the saturation 
of the loops, which does not depend on the in-plane angle and corresponds to the remanence measured when the magnetic field lies along the easy axis direction.

The temperature dependence is shown more clearly in Fig. 2, where the complete spectrum of s-polarized longitudinal Kerr saturation rotation is reported at $300 \mathrm{~K}$ and $12 \mathrm{~K}$ for $\mathrm{Co}_{2} \mathrm{MnGe}$. The main features are well reproduced at both temperatures, except for a slightly higher peak observed at low temperature. This is attributed to a reduced magnetization at high temperature. Similar outcomes are found for the $\mathrm{Co}_{2} \mathrm{MnSn}$ film as well. In the following, we examine in detail the results obtained at room temperature, since the presence of the cryostat windows and the undesirable vibrations of the sample holder lower the signal-to-noise ratio at low temperature, thus preventing an accurate and complete determination of the spectra. It is also worthwhile mentioning that room temperature magnetization measurements, performed with an alternating gradient magnetometer, provide a saturation magnetization corresponding to $83.06 \mathrm{emu} / \mathrm{g}$ and $74.38 \mathrm{emu} / \mathrm{g}$ for and $\mathrm{Co}_{2} \mathrm{MnSn}$, respectively. Not unexpectedly, these values are $20 \%$ and $15 \%$ lower, respectively, than the values obtained at $5 \mathrm{~K}$ on similar films reported previously 13 .

\section{COMPARISON BETWEEN THEORY AND EXPERIMENTS}

In Figs. 3 and 4, the complete experimental and theoretical spectra obtained for longitudinal Kerr geometry are compared for the $\mathrm{Co}_{2} \mathrm{MnGe}$ and $\mathrm{Co}_{2} \mathrm{MnSn}$ films, respectively. The different panels show the MOKE rotation and ellipticity, both in $s$ and $p$ polarization. Our theoretical calculations are obtained according to the following procedure: the $\sigma$ conductivity tensor is calculated by ab-initio simulations. Using Eq. (2), one can compute the Kerr angle at normal incidence on the interface between two semi-infinite media: vacuum and $\mathrm{Co}_{2} \mathrm{MnSn}$ or $\mathrm{Co}_{2} \mathrm{MnGe}$. However, in order to compare the experimental data with the theoretical predictions, the calculated spectrum has to be evaluated in the same geometry and reflection conditions (incident radiation at $45^{\circ}$ on the sample surface) taking also into account the sample structural characteristics. In particular, due to the finite thickness of the Heusler compound, we must consider that the reflected radiation is affected by the optical and magneto-optical response of a multilayered structure. This is possible according to

the formalism introduced by Zak et al. $\underline{\underline{30}}$ : propagation of the radiation within each layer is characterized by a propagation matrix $\mathrm{P}$ and a boundary matrix $\mathrm{A}$, which are functions of 
geometrical parameters, of the complex refractive index $n$ of the constituent material, and the complex magneto-optical coefficient $\mathrm{Q}$ (if the layer is magnetic). The latter terms $(n$, Q) are related to the diagonal and off-diagonal elements, respectively, of the conductivity tensor calculated according to Eq. (1). Using the P and A matrices, the boundary conditions can be directly applied to the electric and magnetic field vectors that, depending on the light polarization ( $s$ or $p$ ), satisfy a different set of homogenous non-linear equations. All results obtained within the Zak model are of the first order in Q. This framework takes into account multiple reflections at the interface between sample layers and it allows us to predict the magneto-optical response of a "film" or a multilayered structure. The wavelength dependence of the refractive index of the sapphire capping layer and substrate was taken from the literature ${ }^{31}$; the vanadium seed layer was neglected since only a small contribution to the reflected radiation is expected from more internal thin layers. For comparison, in the two upper panels of Fig. 3, the results calculated for the semi-infinite magnetic layer ("bulk" calculation) are included only for the case of $s$-wave in $\mathrm{Co}_{2} \mathrm{MnGe}$. We observe that the experimental features (in particular the peak positions) are better matched by the film calculation (solid lines).

The intraband contribution to the conductivity tensor was modeled according to Eq. (3) taking $\hbar \omega_{p}=3.0 \mathrm{eV}$ and $\frac{\hbar}{\tau_{D}}=0.2 \mathrm{eV}$ for both samples, while for the interband term linewidth, the value $\frac{\hbar}{\tau}=0.4 \mathrm{eV}$ was considered. These values were chosen in order to fit the experimental spectra and they are all within the typical range of values for these parameters; their energy dependence was neglected ${ }^{28}$. In particular, the above mentioned value for $\omega_{p}$ differs from the plasma frequency calculated from first principles (i.e. $\hbar \omega_{p} \sim 4.4 \mathrm{eV}$ for both compounds). However, this is not a serious discrepancy since the plasma frequency is known to be strongly affected by sample purity and it is absolutely reasonable (if not expected) that disorder, neglected in the ab-initio simulations, exists in real samples and is responsible for the different $\omega_{p}$ value.

Overall, for both compounds and for both $s$ - and $p$-wave cases, the agreement is good as far as the Kerr rotation and the ellipticity are concerned: theory reproduces the exact sign of the angle and the spectral shape in the whole energy range examined. In particular, the agreement between the energy positions of the main features is excellent, whereas the magnitude shows a disagreement. This is really not too surprising if we consider that: $i$ ) simulations are performed at zero temperature and, therefore, they do not take into account 
spin fluctuations that occur at room temperature as in the case of the actual measurements; ii) at low energies $(\mathrm{E} \leq 1.5 \mathrm{eV})$, intraband excitations play an important role and may not be well reproduced by the simple Drude expression; iii) disorder in the Co and $\mathrm{Mn}$ sublattices occupation might also be present in real samples, thus affecting the electronic and magnetic structure. For example, it has been theoretically shown that Co antisites in bulk $\mathrm{Co}_{2} \mathrm{MnGe}$ have low formation energy and destroy half-metallicity $\underline{32}$, at variance with the NiMnSb case where recent calculations showed that low formation energy defects preserve the half metallic character $\underline{33}$. However, our theoretical investigation allows us to obtain valuable insights on the electronic properties of the materials, which are not easily accessible to experiment alone.

\section{DISCUSSION OF THEORETICAL RESULTS}

Before going into the details of the origin of the optical and magneto-optical properties, we briefly recall the structural and electronic properties of the different Heusler compounds. In particular, in Table \we report our calculated $\underline{\underline{34}}$ equilibrium lattice constant, along with the total magnetic moment, plasma frequency and density of states at the Fermi level $\left(E_{F}\right)$, the last two quantities being spin-resolved. The plasma frequency of the majority-spin component is pretty large for both compounds, reflecting the highly dispersed character of the bands crossing $E_{F}$. We recall that, according to the previously calculated electronic structure for $\mathrm{Co}_{2} \mathrm{MnSn}$ and $\mathrm{Co}_{2} \mathrm{MnGe}^{32}$, the majority spin DOS has metallic character, and the number of unoccupied electronic states is low $(\sim 0.2$ states/(eV cell $))$. On the other hand, the minority spin presents an occupied and unoccupied DOS (obviously separated by the gap giving rise to HM character), both with appreciable weight ( $\sim 5$ states/(eV cell $)$ ). In addition, we observe that, in the energy range of interest (from 0 to $5 \mathrm{eV}$ ), the dipole matrix elements for transitions within the minority channel are more than twice as large than those in the majority spin channel, thus suggesting that optical transitions will more likely occur in the minority spin channel. In the following analysis, therefore, we will mainly consider possible transitions in the minority spin-channel (see below).

Let us first focus on $\mathrm{Co}_{2} \mathrm{MnGe}$. The real parts of the optical conductivity, the dielectric function and the minority band structure are reported in Fig. [5 (a), (b) and (c), respectively. In order to investigate the relation between the features in the optical properties and the 
underlying band structure, we show two spectra obtained with a Lorentzian broadening of $\frac{\hbar}{\tau}=0.4 \mathrm{eV}$ (used in Figs. 3 and 4 to compare with the experimental results) and $0.1 \mathrm{eV}$ (see Eq. (1)), respectively. The higher resolution of the latter spectrum is useful to trace back more clearly the origin of the transitions, starting from the band-structure. In the insets of Fig. 5 (a) and (b), we show a comparison between the total (interband + intraband) and interband-only contributions to the optical properties. The arrows (dotted, solid and dashed) mark some possible vertical transitions with an energy corresponding to the main maxima (at $0.4,2.0$ and $2.5 \mathrm{eV}$, respectively) in the optical conductivity. We note that the optical conductivity shows: $i$ ) a different behavior for energies $<1.5 \mathrm{eV}$, depending on whether the Drude contribution is included or not; $i$ ) three main features in the interband spectra (more evident with the smaller applied broadening), at $\sim 0.4, \sim 2.0$ and $\sim 2.5$ $\mathrm{eV}$. The former consideration is in line with what is generally observed: for typical plasma frequencies of a few eV, the intraband Drude contribution is relevant at small frequencies and becomes negligible at energies larger than $\sim 1.5 \mathrm{eV}$ (given the frequency dependence of Eq. (3) ). Similarly, the dielectric function also shows a typical Drude-like behavior at small energies. In order to better explain the origin of the maxima just mentioned in the real part of the optical conductivity, $\sigma_{x x}$, let us focus on the minority band-structure of $\mathrm{Co}_{2} \mathrm{MnGe}$.

To better understand the hybridization process, we take a look at the minority spin only: the interaction between $d$ orbitals of the two Co atoms in the primitive cell forms bonding (antibonding) states between a 3-fold degenerate $t_{2 g}\left(t_{1 u}\right)$ and 2-fold degenerate $e_{g}$ $\left(e_{u}\right)$ states. The hybridization between $t_{2 g}$ and $e_{g}$ Co-Co orbitals and Mn $d$ states gives rise ${ }^{5}$ to bonding and antibonding levels between 3 -fold $t_{2 g}$ and 2 -fold $e_{g}$, while the $t_{1 u}$ and $e_{u}$ levels do not hybridize with Mn states due to symmetry rules. Therefore, the latter levels are localized at Co sites only. The hybrid level positions can be inferred from the atom projected density of states (not shown); their energies are:

$$
e_{g}^{\downarrow}<t_{2 g}^{\downarrow}<t_{1 u}^{\downarrow}<E_{F}<e_{u}^{\downarrow}<e_{g}^{\downarrow}<t_{2 g}^{\downarrow}
$$

with a total number of occupied minority spin levels equal to 8 electrons per cell. In a more "crude" picture, $\sigma$ can be obtained simply from the product of the dipole matrix element (taken k-independent) and the joined density of states (JDOS) ${ }^{28}$ related to the gradient of the energy of the initial and final states. The highlighted transitions (Fig. 5 panel (c)) at 2.0 and $2.5 \mathrm{eV}$ are relative to excitations from $t_{2 g}^{\downarrow}$ to $e_{u}^{\downarrow}$ and $e_{g}^{\downarrow}$ levels around the $\mathrm{K}$ and $\mathrm{W}$ points 
(and, to a lower extent, around the $\mathrm{X}$ and L points as well) of the Brillouin zone. Transitions around $0.4 \mathrm{eV}$ and $1.0 \mathrm{eV}$ occur close to the $\Gamma$ point and the $\Gamma X$ line exclusively, and involve electronic excitations from $t_{1 u}^{\downarrow}$ to $e_{u}^{\downarrow}$ states. The involved bands show a predominantly $d-$ character and are pretty flat; thus, we expect a larger contribution coming from the JDOS rather than the dipole matrix element.

Finally, since we do not have experimental optical data to compare with, we recall that for the similar compound $\mathrm{Ni}_{2} \mathrm{MnSn}^{36}$, a good agreement between theory and experiments was obtained. More recently, a paper on $\mathrm{Ni}_{2} \mathrm{MnIn}^{37}$ showed that the agreement between firstprinciples simulations and optical measurements was sufficiently good, as far as the main features are concerned, and could be optimized (especially for the peak energy positions), including some empirical corrections (e.g., the $\lambda$-fitting $\frac{38}{3}$ process) to account for many-body and excited state effects. Here, we prefer to not consider any empirical corrections: in fact, since the agreement with the by far more delicate magneto-optical properties is already satisfying, we expect that the inclusion of excited-state corrections would only marginally affect the overall picture which is seen to be accurate enough already within DFT.

Having understood the optical properties, we now move to the magneto-optical properties. Figure 6 (a) shows the calculated polar spectra (both rotation and ellipticity) at normal incidence for bulk $\mathrm{Co}_{2} \mathrm{MnGe}$ and $\mathrm{Co}_{2} \mathrm{MnSn}$ : this is the geometry which provides the highest Kerr response and, therefore, it is the one considered to evaluate the performance of a possible magneto-optical application. The main features of the spectra are similar to those shown in Figs. 3 and 4 , relative to the longitudinal Kerr effect at $45^{\circ}$, except for a much larger peak size. First of all, the longitudinal Kerr rotation is around $0.05^{\circ}$ (although it should be recalled that the maximum Kerr rotation strictly depends on the smearing value considered): this is a typical value for $3 d$ based materials, which are generally not very active from the magneto-optical point of view. Upon reduction of the broadening values within a physically meaningful range (i.e. $\frac{\hbar}{\tau}=0.1 \mathrm{eV}$ ), the Kerr angle increases up to $0.07^{\circ}$. Still, given the $3 d$ nature of the compounds, these are not unexpected results, at variance with the case of PtMnSb where a giant Kerr rotation was observed both experimentally $\underline{18}$ and theoretically $\underline{\underline{19}}$. This is consistent with the picture given previously $\underline{\underline{19}} \underline{\underline{39}}$ for half-Heusler compounds: in order to have an extremely high Kerr angle, a large spin-orbit coupling is required in addition to a large magnetic moment, in this case provided by Mn atoms.

In our samples, cobalt (a light transition metal) acts as a "cation", providing a spin-orbit 
coupling much smaller than other heavier elements (such as Pt or Au). On the other hand, also the $p$ element (Ge or $\mathrm{Sn}$ ) behaves differently from $\mathrm{Sb}$, but the effect of an increased spinorbit coupling with increased atomic number of the $p$-electron source element is negligible ${ }^{32}$, since most of the states involved in the transitions are localized on the cation sites (Co, Mn). Our generally small Kerr angles indicate that half-metallicity alone (such as that typical of $\mathrm{CO}_{2} \mathrm{MnSn}$ and $\mathrm{CO}_{2} \mathrm{MnGe}$ ) is not sufficient to guarantee strong $\mathrm{MO}$ effects. Along the same line, the maximum magnitude of the Kerr angle in $\mathrm{Co}_{2} \mathrm{MnSn}$, which deviates from half metallicity, is not remarkably smaller, but rather of the same order of magnitude as in $\mathrm{Co}_{2} \mathrm{MnGe}$. Moreover, irrespective of the actual chemical composition, the Kerr spectra look pretty similar for the two compounds: the Kerr rotation is generally negative and shows two minima, marked with vertical lines in Fig. 6, whereas the Kerr ellipticity exhibits some zero crossings and two main positive peaks. This has to be related, of course, to the underlying electronic structure which, as noted before, is very similar in the two compounds.

As shown in Eq. (2), the Kerr angle is given by a strictly magneto-optical numerator, which depends only on the off-diagonal part of the $\sigma$ matrix (and, therefore, is related to the simultaneous presence of exchange and spin-orbit coupling), and by an optical denominator (related to the diagonal part of the conductivity tensor only). Therefore, some hints about the origin of the features in the Kerr angles can be gained by plotting, as reported in Fig. 6. the Kerr rotation (panel (a)) and, separately, the numerator (real and imaginary parts, panel (b)) and inverse denominator (real and imaginary parts, panel (c)) of Eq. (2). In agreement with what was suggested for the half-Heusler compounds $\frac{19}{}$ and also confirmed for a large class of full-Heusler alloys, we find that the first minimum (photon energy $\sim 1.0$ $\mathrm{eV}$ ) is mostly due to optical contributions (compare with panel (b)), whereas the second minimum $(\sim 3.4 \mathrm{eV})$ shows a magneto-optical origin (compare with panel $(\mathrm{c})$ ).

Finally, we recall that the imaginary part of the off-diagonal conductivity (Fig. 6(c)) is directly related to the difference in absorption of the left- (LCP) and right-circularly polarized (RCP) light. Therefore, the wavelengths characterized by a zero rotation angle (though slightly dependent on the broadening used) mark the points where LCP and RCPinduced transitions have the same overall probability. For both $\mathrm{Co}_{2} \mathrm{MnGe}$ and $\mathrm{Co}_{2} \mathrm{MnSn}$, this occurs around 2.0-2.3 eV. 


\section{CONCLUSIONS}

In summary, we have presented a full characterization of MOKE spectra in $\mathrm{Co}_{2} \mathrm{MnGe}$ and $\mathrm{Co}_{2} \mathrm{MnSn}$ Heusler compounds, both from first-principles and experimental points of view. We have shown that, for a correct comparison between theory and experiment, the exact measurement conditions have to be taken into account, namely: Kerr geometry, angle of incidence of the light and multilayered structure of the samples. This has been achieved by implementing ab-initio simulations in DFT of the tensor $\sigma$ within the Zak model which imposes the correct boundary conditions in any given geometry and multilayered configuration. The maximum Kerr rotation results to be of the order of $\sim 0.25^{\circ}$, which is not very appealing for magneto-opto-electronic applications. On the other hand, the agreement between theory and experiment allowed a deep understanding of the main features in the optical and magneto-optical spectra of these compounds in terms of the underlying bandstructure. This could be very useful in the design of ad-hoc materials, since a solid basis for predicting their properties and their possible applications is established.

\section{Acknowledgments}

Work at Northwestern University supported by the U.S.N.S.F. through its MRSEC Program at the N. U. materials Research Center.

1 T. Dietl, H. Ohno, F. Matsukura, J. Cibert and D. Ferrand, Science 287, 1019 (2000); Y. Ohno, D. K. Youn, B. Beschoten, F. Matsukura, H. Ohno and D. D. Awschalom, Nature 402, 790 (1999).

2 R. Fiederling, M. Keim, G. Reuscher, W. Ossau, bi G. Schmidt, A. Waag and L. W. Molenkamp, Nature 402, 787 (1999).

3 R. A. De Groot, F. M. Mueller, P. G. Van Engen and K. H. J. Buschow, Phys. Rev. Lett. 50, 2024 (1983).

4 I. Galanakis, P. Mavropoulos and P. H. Dederichs, J. Phys. D: Appl. Phys. 39, 765 (2006).

5 I. Galanakis and P.H. Dederichs, Half-metallic Alloys - Fundamentals and Applications, Lecture Notes in Physics Vol. 676, Springer, Berlin Heidelberg, p. 1 (2005). 
6 S. Picozzi, A. Continenza and A.J. Freeman, Phys. Rev. B 66, 094421 (2002).

7 T. Ambrose, J. J. Krebs, and G. A. Prinz, Appl. Phys. Lett. 76, 3280 (2000).

8 M. P. Raphael, S. F. Cheng, B. N. Das, B. Ravel, B. Nadgorny, G. Trotter, E. E. Carpenter and V. G. Harris, MRS Proceedings, Spring meeting, 2001.

9 T. Ambrose, J. J. Krebs and G. A. Prinz, J. Appl. Phys. 87, 5463 (2000).

10 J. W. Dong, L. C. Chen, C. J. Palmstrøm, R. D. James, and S. McKernan, Appl. Phys. Lett. 75, 1443 (1999).

11 J. W. Dong, L. C. Chen, J. Q. Zie, T. A. R. Muller, D. M. Carr, C. J. Palmstrom, S. McKernan, Q. Pan and R. D. James, J. Appl. Phys. 88, 7357 (2000).

12 J. W. Dong, J. Lu, J. Q. Xie, L. C. Chen, R. D. James, S. McKernan, and C. J. Palmstrøm, Physica E 10, 428 (2001).

13 U. Geiersbach, A. Bergmann, K. Westerholt, Thin Solid Films 425, 225 (2003).

14 K. Westerholt, A. Bergmann, J. Gabris, A. Nefedov and H. Zabel, Half-metallic Alloys - Fundamentals and Applications, Lecture Notes in Physics Vol. 676, Springer, Berlin Heidelberg, p. 67 (2005).

15 X. Y. Dong, C. Adelmann, J. Q. Xie, C. J. Palmstrøm, X. Lou, J. Strand, P. A. Crowell, J.-P. Barnes, and A. K. Petford-Long, Appl. Phys. Lett. 86, 102107 (2005).

16 S. Fujii, S. Sugimura, S. Ishida and S. Asano, J. Phys.: Condens. Matter 2, 8583 (1990); S. Ishida, S. Fujii, S. Kashiwagi and S. Asano, J. Phys. Soc. Japan 64, 2152 (1995).

17 S. Ishida, T. Masaki, S. Fujii, S. Asano, Physica B 245, 1 (1998).

18 P.G. Van Engen, K. H. J. Buschow, R. Jonegreur, and M. Erman, Appl. Phys. Lett. 42, 202 (1983).

19 V. N. Antonov, A. N. Yaresko, A. Ya. Perlov, V. V. Nemoshkalenko, P. M. Oppeneer, and H. Eschrig, Low Temp. Phys. 25, 387 (1999).

20 W. H. Wang, M. Przybylski, W. Kuch, L. I. Chelaru, J. Wang, Y. F. Lu, J. Barthel, H. L. Meyerheim, and J. Kirschner, Phys. Rev. B 71, 144416 (2005).

21 K. Miyamoto, A. Kimura, K. Iori, K. Sakamoto, T. Xie, T. Moko, S. Qiao, M. Taniguchi, K. Tsuchiya, J. Phys.: Condens. Matter 16, S5797 (2004).

22 A. Nefedov, J. Grabis, A. Bergmann, K. Westerholt, H. Zabel, Physica B 345, 250 (2004).

23 J. Grabis, A. Bergmann, A. Nefedov, K. Westerholt, and H. Zabel, Phys. Rev. B 72, 024437 $(2005)$. 
24 E. Wimmer, H. Krakauer, M. Weinert and A. J. Freeman, Phys. Rev. B 24, 864 (1981).

25 H.J. Monkhorst and J.D. Pack, Phys. Rev. B 13, 5188 (1976).

26 K. H. J. Buschow, Ferromagnetic Materials, edited by E. P. Wohlfarth and K. H. J. Buschow (North-Holland, Amsterdam, 1988), Vol. 4, p. 493.

27 C. S. Wang and J. Callaway, Phys. Rev. B 9, 4897 (1974).

28 H. Ebert, Rep. Prog. Phys. 59, 1665 (1996).

29 M. Kim, A. J. Freeman and R. Wu, Phys. Rev. B 59, 9432 (1999).

30 J. Zak, E.R. Moog, C. Liu and S.D. Bader, J. Magn. Magn. Mater. 89, 107 (1990).

31 P. D. Edward, Handbook of optical constants of solids II, Academic Press (1991).

32 S. Picozzi, A. Continenza, and A. J. Freeman, Phys. Rev. B 69, 094423 (2004).

33 B. Alling, S. Shallcross, I. A. Abrikosov, Phys. Rev. B 73, 064418 (2006).

34 The lattice constants were calculated within the Generalized Gradient Approximation according to Perdew-Becke-Erzenhof parametrization, which is known to give lattice constants close to experiments.

35 I. Galanakis, P. H. Dederichs, and N. Papanikolaou, Phys. Rev. B 66, 174429 (2002); Phys. Rev. B 66, 134428 (2002).

36 M. M. Kirillova, Yu. I. Kuzmin, Yu. V. Knyazev, E. I. Shreder, Phys. Met. Metallogr. 83, 590 (1997).

37 Y. V. Kudryavtsev, Y. P. Lee and J. Y. Rhee, Phys. Rev. B 69, 195104 (2004).

38 J. Y. Rhee, B. N. Harmon and D. W. Lynch, Phys. Rev. B 55, 4124 (1997).

39 L. Offernes, P. Ravindran and A. Kjekshus, Appl. Phys. Lett. 82, 2862 (2003).

40 P.J. Webster and K.R.A. Ziebeck, Alloys and compounds of d-elements with main group elements Part 2, Springer-Verlag, Berlin (1988). 


\begin{tabular}{|c|c|c|c|c|c|c|}
\hline \hline & $\begin{array}{c}a \\
(\AA)\end{array}$ & $\begin{array}{c}\mu_{\text {tot }} \\
\left(\mu_{B}\right)\end{array}$ & $\begin{array}{c}\hbar \omega_{p}^{\uparrow} \\
(\mathrm{eV})\end{array}$ & $\begin{array}{c}\hbar \omega_{p}^{\downarrow} \\
(\mathrm{eV})\end{array}$ & $\begin{array}{c}D^{\uparrow}\left(E_{F}\right) \\
(\text { states} / \mathrm{eV})\end{array}$ & $\begin{array}{c}D^{\downarrow}\left(E_{F}\right) \\
(\text { states } / \mathrm{eV})\end{array}$ \\
\hline \hline $\mathrm{Co}_{2} \mathrm{MnGe}$ & 5.74 & 5.00 & 4.4 & 0.0 & 1.5 & 0.0 \\
\hline $\mathrm{Co}_{2} \mathrm{MnSn}$ & 6.00 & 5.03 & 4.4 & 0.7 & 0.9 & 0.1 \\
\hline
\end{tabular}

TABLE I: Relevant calculated properties of $\mathrm{Co}_{2} \mathrm{MnGe}$ and $\mathrm{Co}_{2} \mathrm{MnSn}$ Heusler compounds: equilibrium lattice constant, total magnetic moment per cell $\left(\mu_{t o t}\right)$, plasma frequency for up and down spin channels $\left(\omega_{p}^{\uparrow}\right.$ and $\left.\omega_{p}^{\downarrow}\right)$, and their density of states at $E_{F}\left(D\left(E_{F}\right)^{\uparrow}\right.$ and $\left.D\left(E_{F}\right)^{\downarrow}\right)$.

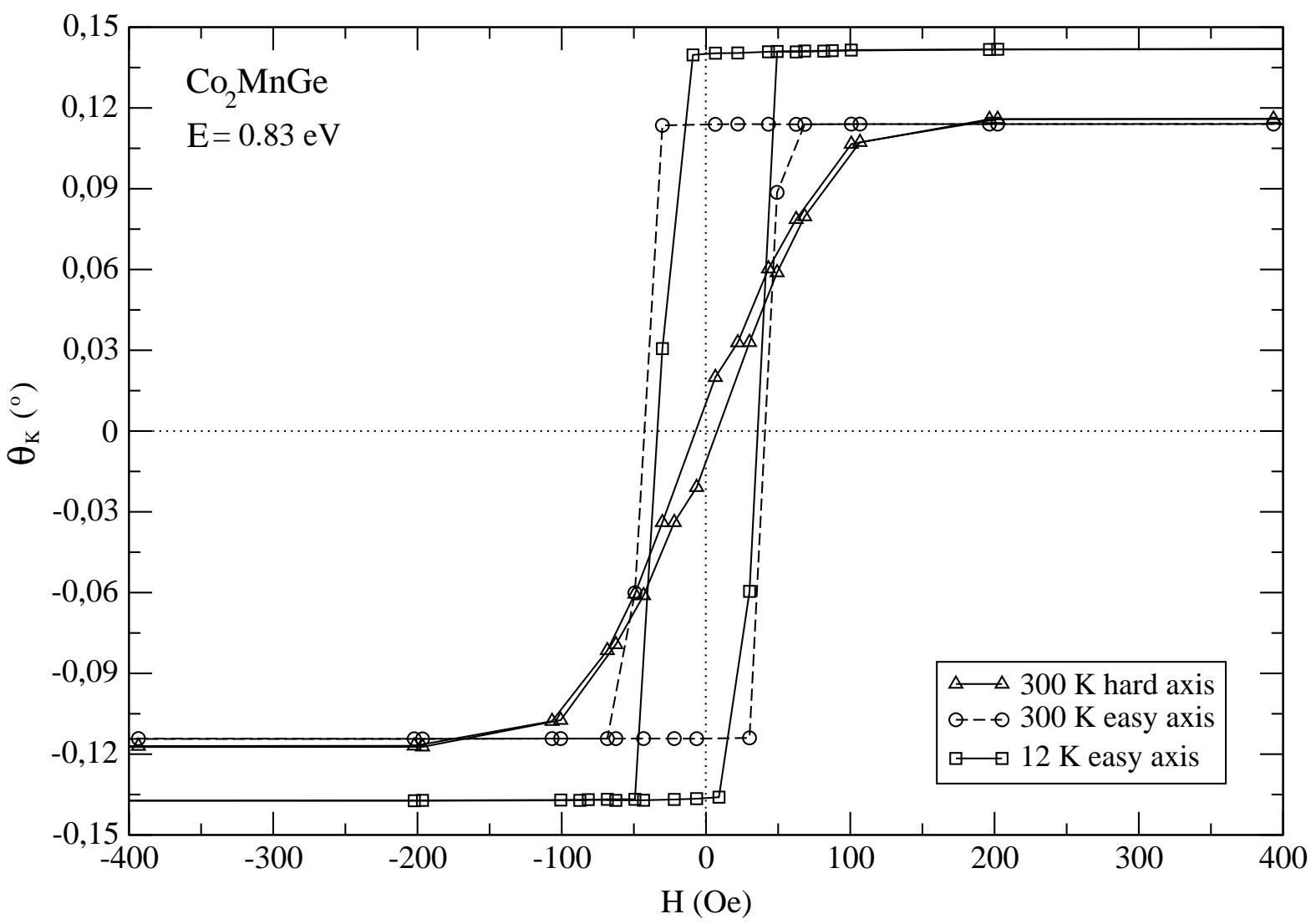

FIG. 1: $s$-polarized longitudinal MOKE rotation hysteresis loops of $\mathrm{Co}_{2} \mathrm{MnGe}$ at photon energy $=$ $0.83 \mathrm{eV}$. Both easy and hard directions of magnetization are measured at room temperature. 


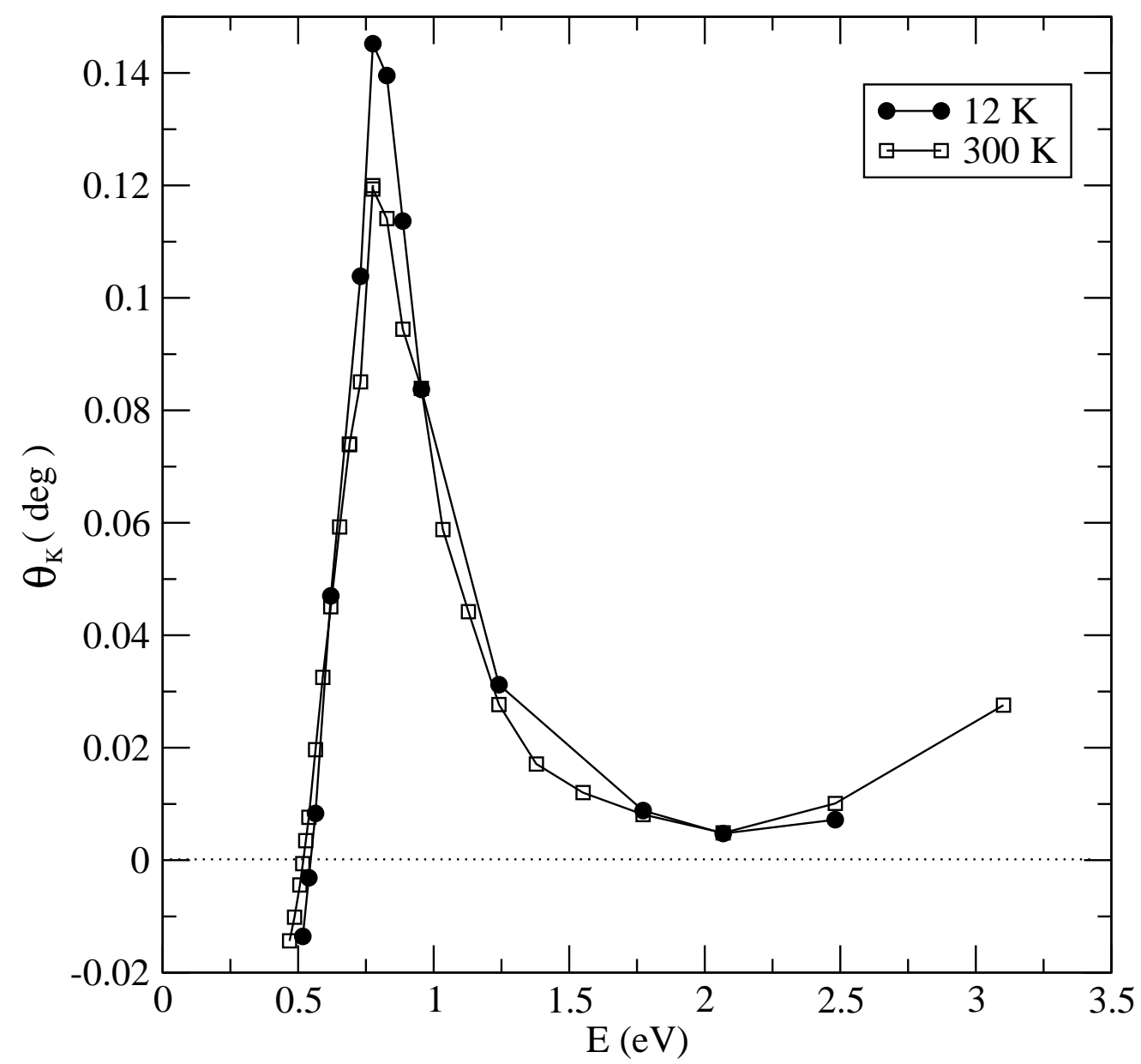

FIG. 2: s-polarized longitudinal Kerr rotation at saturation as a function of photon energy at two different temperatures for $\mathrm{Co}_{2} \mathrm{MnGe}$. 
s-wave
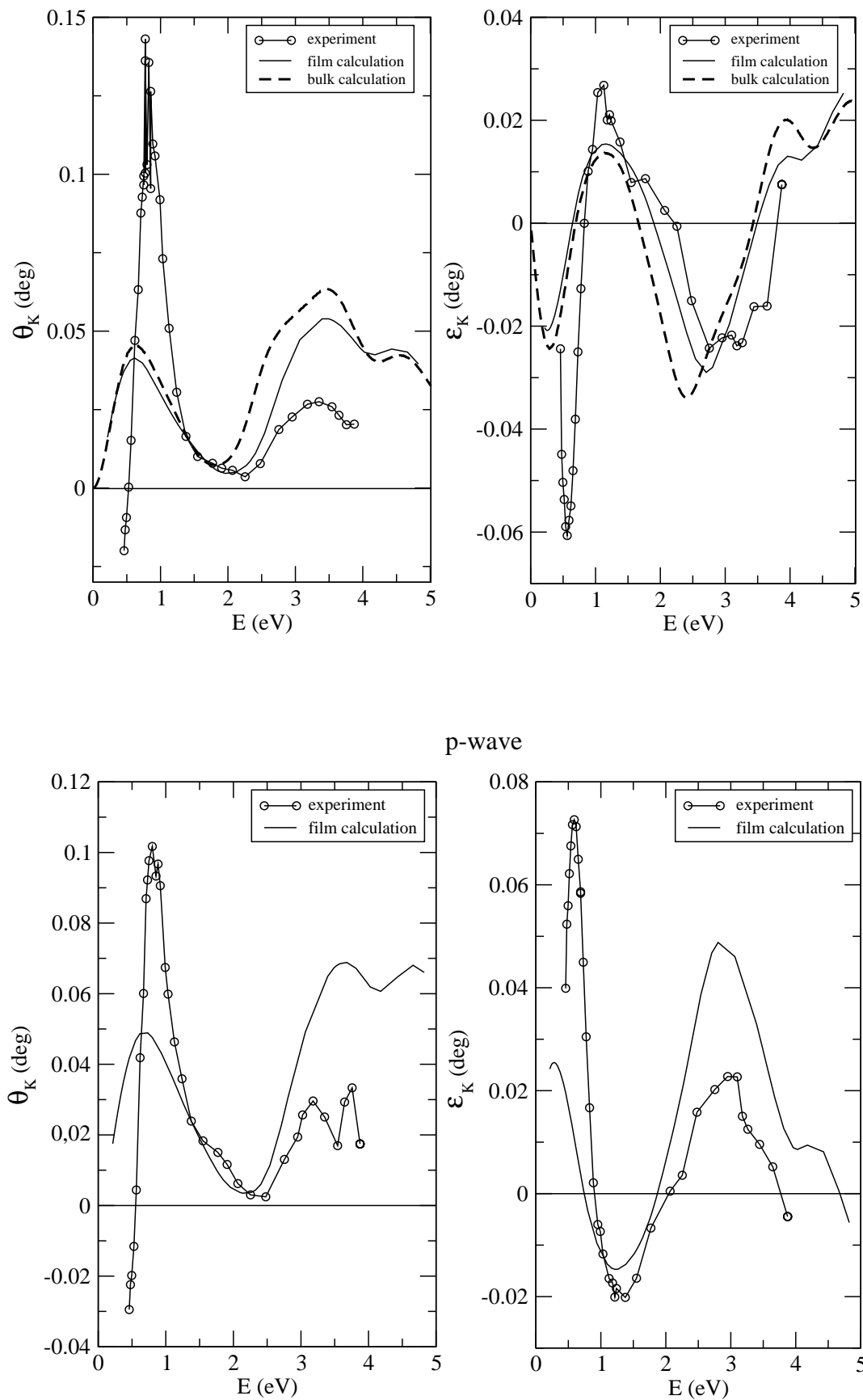

p-wave

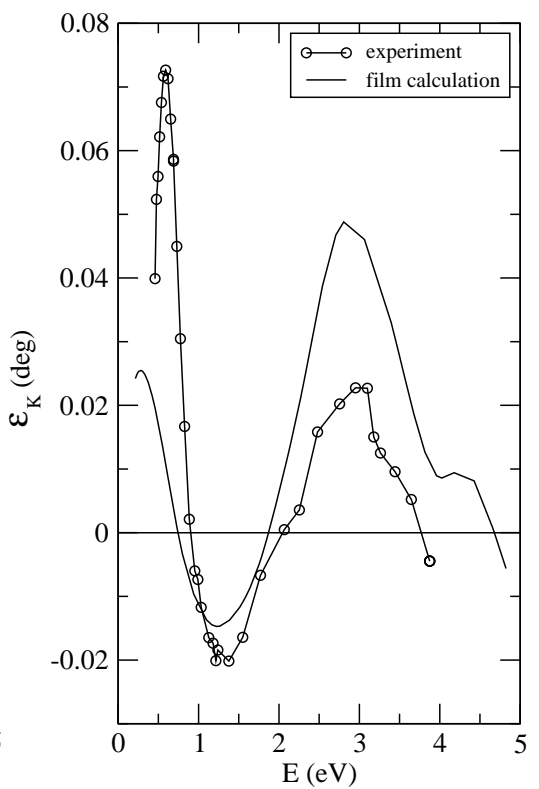

FIG. 3: Theoretical and experimental longitudinal Kerr spectra as a function of photon energy in $\mathrm{Co}_{2} \mathrm{MnGe}$. Top panels (s-wave) report both bulk (dashed) and $100 \mathrm{~nm}$ thick film (solid) calculations. 
s-wave
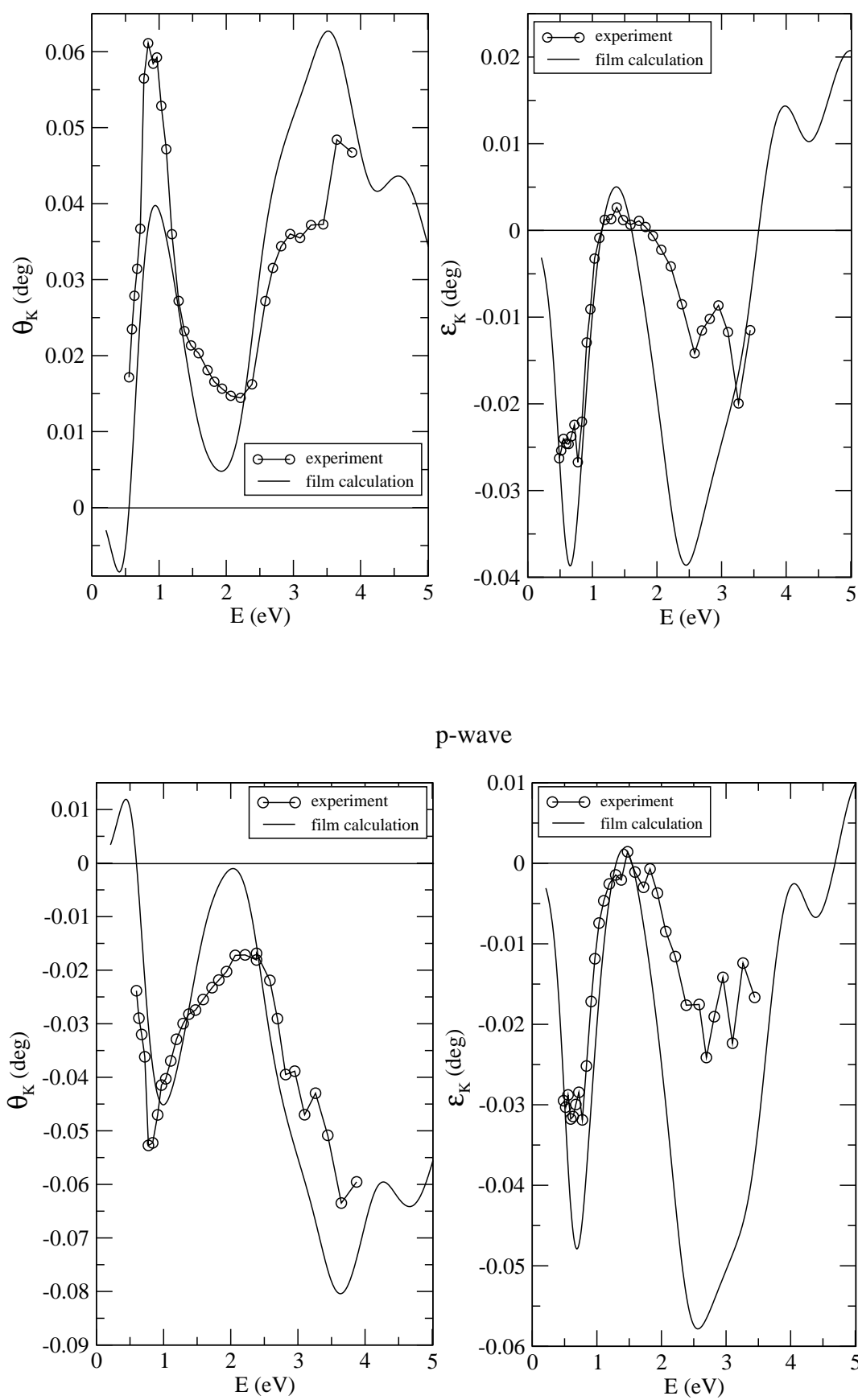

p-wave

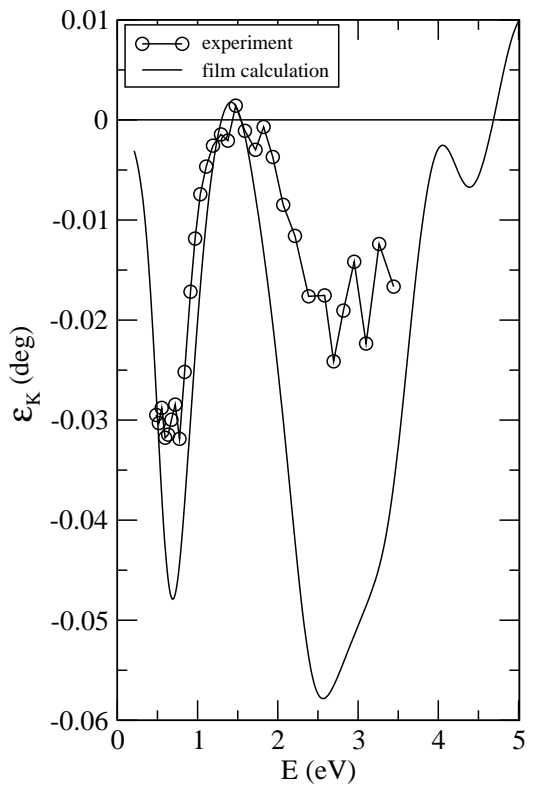

FIG. 4: Theoretical and experimental longitudinal Kerr spectra for $\mathrm{Co}_{2} \mathrm{MnSn}$ as a function of the photon energy in both polarizations of the incident radiation. 


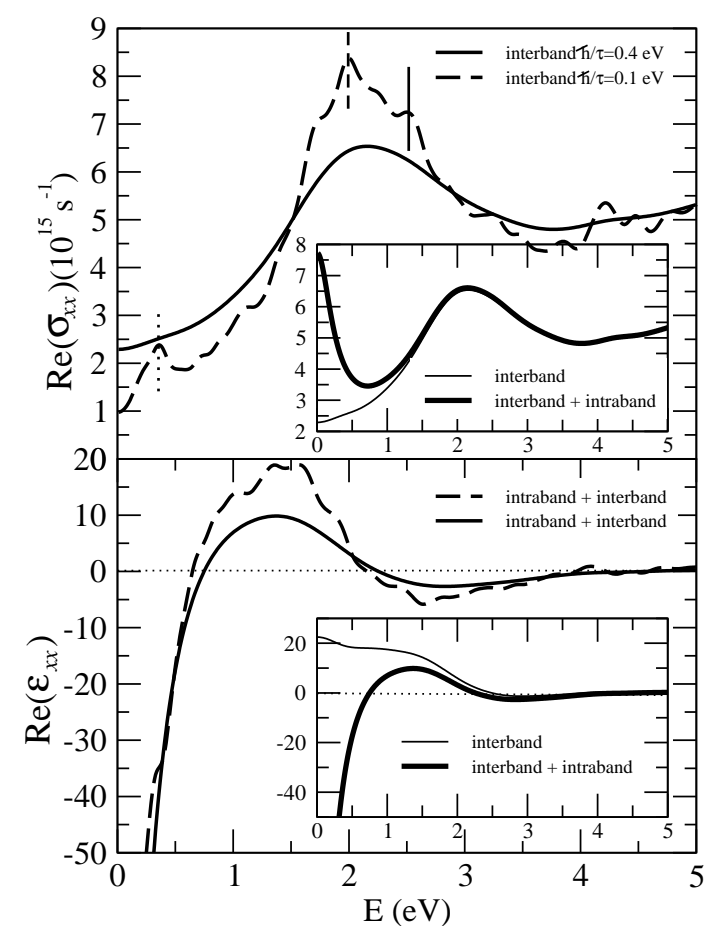

(a)

(b)

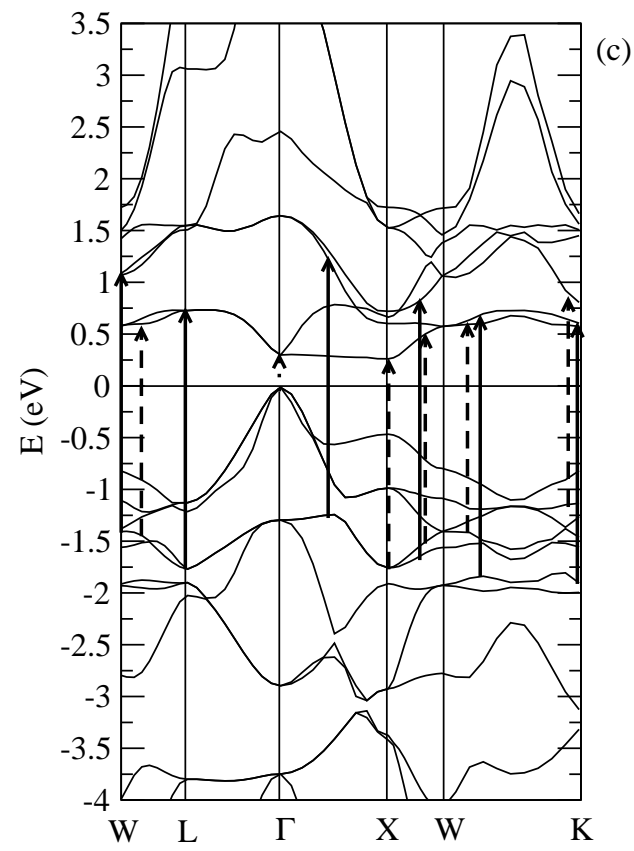

FIG. 5: Optical properties of $\mathrm{Co}_{2} \mathrm{MnGe}$ : (a) real parts of optical conductivity and (b) dielectric function for different broadenings: $\frac{\hbar}{\tau}=0.1 \mathrm{eV}$ (dashed) and $\frac{\hbar}{\tau}=0.4 \mathrm{eV}$ (solid). The insets in (a) and (b) show the effect of the Drude contribution $\left(\frac{\hbar}{\tau}=0.4 \mathrm{eV}, \hbar \omega_{p}=3.0 \mathrm{eV}\right.$ and $\left.\frac{\hbar}{\tau_{D}}=0.2 \mathrm{eV}\right)$. (c) Minority band structure of $\mathrm{Co}_{2} \mathrm{MnGe}$ : the arrows (dotted, dashed and solid) mark the transitions responsible for the main features highlighted in the optical conductivity, panel (a), at 0.4, 2.0 and $2.5 \mathrm{eV}$, respectively. The zero of the energy scale is set to the Fermi level. 

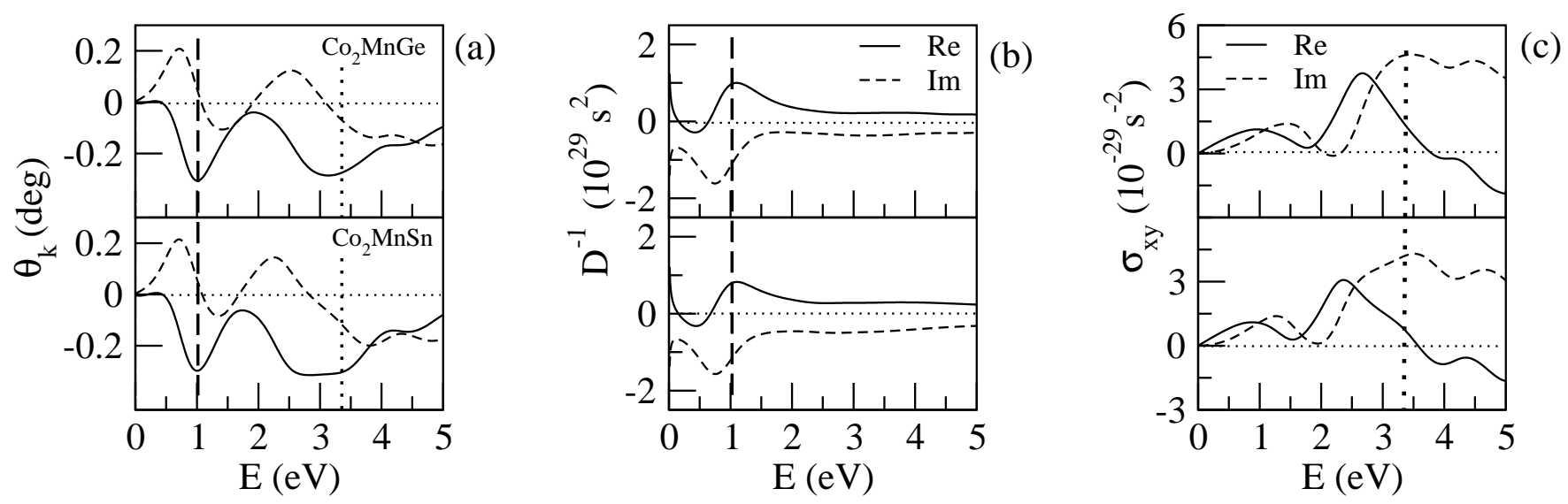

FIG. 6: (a) Polar Kerr rotation (solid curve) and ellipticity (dashed curve) at normal incidence in $\mathrm{Co}_{2} \mathrm{MnGe}$ (top panel) and $\mathrm{Co}_{2} \mathrm{MnSn}$ (bottom panel). (b): inverse denominator in the complex Kerr angle of Eq. (2). (c): off-diagonal conductivity (see numerator of Eq. (2)). In (b) and (c), solid and dashed curves denote real and imaginary parts, respectively. 